\title{
Expanding lipidome coverage using MS/MS-aided untargeted data-independent RP-UPLC-TOF-MSE acquisition
}

\author{
Panagiotis A Vorkas*,1 \\ ${ }^{1}$ Section of Biomolecular Medicine, Division of Computational \& Systems Medicine, Department of Surgery \& Cancer, Faculty of \\ Medicine, Imperial College London, Sir Alexander Fleming Bldg., South Kensington Campus, London SW7 2AZ, UK \\ * Author for correspondence: Tel.: +44 0207594 5309; p.vorkas@imperial.ac.uk
}

Lipid function and importance in disease are being rediscovered due to modern advancements in chemical analysis. RP-UPLC-TOF-MS is now the lipidomics tool of choice and can provide the demanded specificity for detecting the great diversity of the lipidome. It can offer simplicity, rapidity, robustness and high throughputness, without the need for further optimization in current sample preparation protocols. This method can cover the major lipid categories with the ability to detect several corresponding subclasses. It can deliver adequate information for deciphering fatty chain length, unsaturation and regioisomerism. It has enabled the detection of a vast number of lipids, of which more than 250 are reported here. These lipids were detected from applications in a variety of biological matrices and species.

First draft submitted: 14 November 2017; Accepted for publication: 16 January 2018; Published online: 1 March 2018

Keywords: data-dependent acquisition $\bullet$ lipidomics $\bullet \mathrm{MS}^{\mathrm{E}}$ data-independent acquisition $\bullet$ untargeted analysis

Lipids are broadly defined as organic molecules of biological origin that are soluble in organic solvents. Subsequently, a great range of molecular classes and subclasses fall under this description. In biological systems and especially mammals, lipid structure can further be altered in response to diet constituents and interacting microorganisms. Consequently, biochemical reactions producing lipid structures, can go beyond the control of specific native genes [1]. This vast diversity of lipids, along with their high lipophilicity, renders their analysis in biological samples very challenging.

Beyond energy storage and membrane formation, lipids can function as vital signaling mediators in biological systems. Furthermore, alterations in the consistency of the lipids and their incorporating fatty chains in the cell membrane, can result to changes in permeability with consequent biological implications [2]. Therefore, lipid molecules can have a pivotal role in maintaining homeostasis and development of several diseases [3,4].

The lipid classes are broadly divided in eight categories: fatty acyls, glycerolipids, glycerophospholipids, sphingolipids, sterol lipids, prenol lipids, saccharolipids and polyketides [5]. These categories are subsequently divided into several classes and subclasses. In the past, lipid subclasses were integrally studied without consideration of their incorporated fatty chains. However, modern advancements in instrumentation and bioanalytical approaches have altered the framework for which lipid molecules are now studied. It is nowadays clearly understood that biological properties of intrasubclass lipid species can differ, depending on the incorporated fatty chains.

Reversed-phase (RP) chromatography has now become the liquid chromatography method of choice in lipid analysis, due to the enhanced intrasubclass separation ability. It replaces the utilization of normal phase, which was preferred in previous years due to the ability to provide enhanced intersubclass separation. This, however, can lead to analyte coelution and ion suppression phenomena. Nonetheless, the use of RP chromatography has been supported by recent technological advances. These include the ability of columns and solvent-managing systems to endure high backpressure, but also the ability for application of high column temperatures. These new advancements have provided the capability to employ mobile phase solvents of high viscosity, such as isopropanol, improving the ability to manipulate the partitioning of analytes of high lipophilicity. 
The superior performance of modern UPLC-MS instrumentation, along with advances in informatics, have also made the robust use of untargeted approaches possible. Untargeted lipidomics can provide the advantage of detecting lipid structures that have not been previously encountered. Additionally, factors exogenous to the studied biological system can drastically alter the lipidome, producing molecular structures that would not otherwise be expected or predicted in the specific system. There are numerous examples where untargeted approaches have delivered such molecules, including the discovery of branched fatty acid (FA) esters of hydroxyl-fatty acids in relation to type II diabetes [6], the N,N-dimethylsphingosine involvement in chronic neuropathic pain [7] and the implication of ceramide-phosphoethanolamines in atherogenesis [8].

Here, by using an untargeted RP-UPLC-TOF-MS ${ }^{\mathrm{E}}$ method, the relative quantitation of a comprehensive range of lipid molecules, from a wide range of classes and subclasses, is achieved. This method is highly versatile, as it does not require any specific adjustments to current sample preparation protocols and can be employed after any generic lipid extraction method. A representative catalog of more than 250 lipid species, robustly determined by the described method and in a variety of matrices, is demonstrated. The powerful capabilities of this method are shown by the enhanced separation performance for intrasubclass lipid species and isomers, elevated specificity, ability to detect unknown molecules with high precision and enhanced lipidome coverage. The $\mathrm{MS}^{\mathrm{E}}$ approach allows for the acquisition of two parallel alternating scans, at low and high collision energy [9]. In this mode, information about both precursor ions and high collision energy fragments can be collected simultaneously. Most importantly, this approach is data-independent, whereas no specific ion is selected for fragmentation, making it very attractive for untargeted pipelines. Furthermore, by using an optimized lipid extraction protocol [10], the described untargeted lipidomics workflow has been successfully employed in a range of biological matrices, such as blood serum and plasma [11-13], cells, a variety of tissue types (healthy and diseased) from humans, rodents, bovines and ovines (liver, brain, breast, adipose, arteries $[8,14,15]$, veins $[10,16,17]$, atherosclerotic plaques $[8,14,15]$, tissue segments from the GI tract, skeletal muscle and heart) and model organisms such as Caenorhabditis elegans.

\section{Method}

Samples

The employed methodology was applied in a variety of samples, such as human/rodent blood serum and plasma, cells, model organisms such as C. elegans, and a variety of tissues including liver, brain, breast (normal and cancerous), adipose, arteries and veins, atherosclerotic plaques, tissue segments from the GI tract, skeletal muscle and heart. These sample types were obtained from humans, rodents, bovines and ovines.

\section{Sample preparation}

Organic phase extraction was performed using an optimized protocol $[10,18]$. It included addition of an organic solvent mixture of methyl tert-butyl ether/methanol (3:1); bead-beating for tissue and $C$. elegans and vortexing for plasma/serum and cells. This was followed by centrifugation, decanting of the supernatant and evaporation in a vacuum concentrator. Samples were stored and approaching analysis were reconstituted in isopropanol (IPA)/acetonitrile (ACN)/water (3:1:1), followed by vortexing, centrifugation and decanting of the supernatant in LC-MS vials.

\section{UPLC-MS analysis}

A detailed protocol of the UPLC-MS materials and instrumentation used has been recently described [15,18]. Briefly, a 20 min gradient mobile phase system was employed using a UPLC binary solvent manager. The mobile phase A consisted of ACN/water (60:40), $10 \mathrm{mM}$ ammonium formate and $0.1 \%$ formic acid and mobile phase B of IPA/ACN (90:10) and 0.1\% formic acid (FA). The gradient profile was as follows: $60-57 \% \mathrm{~A}(0.0-2.0 \mathrm{~min})$, $57-50 \%$ A (2.0-2.1 min; curve 1), 50-46\% A (2.1-12.0 min), 46-30\% A (12.0-12.1 min; curve 1), 30-1\% A $(12.1-18 \mathrm{~min}), 1-60 \% \mathrm{~A}(18.0-18.1 \mathrm{~min})$ and $60 \% \mathrm{~A}(18.1-20.0 \mathrm{~min})$. The flow rate was set at $0.4 \mathrm{ml} / \mathrm{min}$. An Acquity UPLC CSH C18, $2.1 \times 100$ mm, $1.7 \mu \mathrm{m}$ column (Waters Corp, MA, USA) was used for chromatographic separation and maintained at $55^{\circ} \mathrm{C}$. The chromatographic separation was followed by detection using an ESI-TOFMS. Two parallel alternating scans were employed for low collision energy and high collision energy acquisition. The high collision energy scan was performed without ion selection $\left(\mathrm{MS}^{\mathrm{E}}\right.$ channel) [9]. 


\section{Data processing}

Data were processed in R using the open access XCMS package [19]. Data processing consisted of peak picking ('centWave' algorithm), retention time correction ('obiwarp' algorithm), peak grouping ('density' algorithm) and filling of zero intensity features with the regional background noise ('fillPeaks' algorithm).

\section{Normalization \& use of internal standards}

Data were assessed for the most appropriate normalization method, based on the profiles of the QCs (repeatability), dilution series (intensity response according to dilution factor) and samples (intensity drift; grouping of positive controls). Normalization was performed using total area or median fold change normalization. There were occasions where normalization was not necessary. Furthermore, normalization using internal standards was preferred and was typically employed when a lipid (sub-)class was of special interest. Heavily labeled and nonlabeled internal standards that have been used were the following: 1,2-diheptadecanoyl-sn-glycero-3-phosphorylcholine $(25 \mu \mathrm{g} / \mathrm{ml}$; for phosphatidylcholines), heptadecanoic acid (methyl-d3) $(250 \mu \mathrm{g} / \mathrm{ml}$; for FAs), 1,3(d5)-diheptadecanoyl-2-(10Zheptadecenoyl)-glycerol $(25 \mu \mathrm{g} / \mathrm{ml}$; for triacylglycerols $), 18: 1$ cholesteryl-d7 ester $(100 \mu \mathrm{g} / \mathrm{ml}$; for cholesteryl esters $)$ and cholest-5-en-3ß-ol(d7) $(100 \mu \mathrm{g} / \mathrm{ml}$; for unconjugated sterols).

\section{Lipid structure assignment}

For lipid structure assignment, accurate $\mathrm{m} / \mathrm{z}$ measurements were matched to the accurate mass of lipids from online MS databases (METLIN [20], HMDB [21] and LIPID MAPS [22]). After an assessment of retention time and isotopic pattern, tandem MS spectra (UPLC-MS ${ }^{\mathrm{E}}$ and UPLC-MS/MS) were employed for comparison to spectra available in online databases and literature (where available). If authentic standards were commercially available, unknown lipids were matched to accurate mass measurements, retention time and MS/MS spectra of the corresponding standard, under identical experimental conditions (within-day experiment). In Supplementary Table 1, the level of assignment for each identified lipid species is depicted.

\section{Advantages of the use of RP-UPLC}

The RP-UPLC maintains the ability to separate by lipid class, as demonstrated in Figure 1. Subclass separation does not demonstrate distinct coeluting regions from lipids of the same subclass. However, this provides the advantage of enhanced separation for individual lipid species and reduces ion suppression/enhancement phenomena, due to less analyte coelution. The use of the UPLC technique is well suited for untargeted applications, as it can provide enhanced sensitivity [23] and provide detection of molecules known to be present in low concentrations in biological matrices [8].

Importantly, RP-UPLC can achieve separation between isomers, that can differ in the glycerol carbon position to which the fatty chain is esterified to ( $s n-1$ and $s n-2$ positions), translated into important biological information. Lipid subclasses including lysophosphatidylcholines, lysophosphatidylethanolamines and lysophosphatidylinositols can achieve such chromatographic separation (Figure 2). Geometric isomers can also be resolved with the employed chromatography, with examples from the free FA chain subclass, such as the demonstrated separation between oleic (cis) and elaidic (trans) acids and linoleic (cis, cis) and linoelaidic (trans, trans) acids [15]. This has been further demonstrated in glycerophospholipids and specifically in the phosphatidylglycerol class [15]. The use of IPA in the mobile phase solvent system proved capable for enhancing chromatographic performance and elution of highly lipophilic molecules such as triacylglycerols (Figure 1).

\section{Advantages of the use of $\mathrm{MS}^{\mathrm{E}}$ data-independent acquisition in untargeted lipidomics}

The $\mathrm{MS}^{\mathrm{E}}$ approach can prove to be a valuable, informative tool in terms of molecular structure elucidation in lipidomics applications. In fact, the information acquired by collision induced dissociation data-independent techniques, such as UPLC-MS ${ }^{\mathrm{E}}$, can go beyond assisting with molecular structure assignment $[15,24]$. The produced high collision energy specific fragments can be characteristic of molecular components such as lipid head-groups and fatty acyl chains of lipid species. Therefore, the parallel acquisition of low and high collision energy scans allows for (semi-)quantification of the characteristic fragments and subsequent molecule of derivation, with higher specificity. The use of UPLC-MS ${ }^{\mathrm{E}}$ can also maximize the instrument duty cycle providing enhanced sensitivity of fragment ions [9]. Furthermore, the data-independent mode of the UPLC-MS ${ }^{\mathrm{E}}$ requires no prior knowledge for selecting an ion for application of high collision energy, as opposed to data-dependent acquisition (DDA), in which the MS instrument switches from full-scan MS mode to MS/MS. However, the latter can result both in a loss of 
(A)

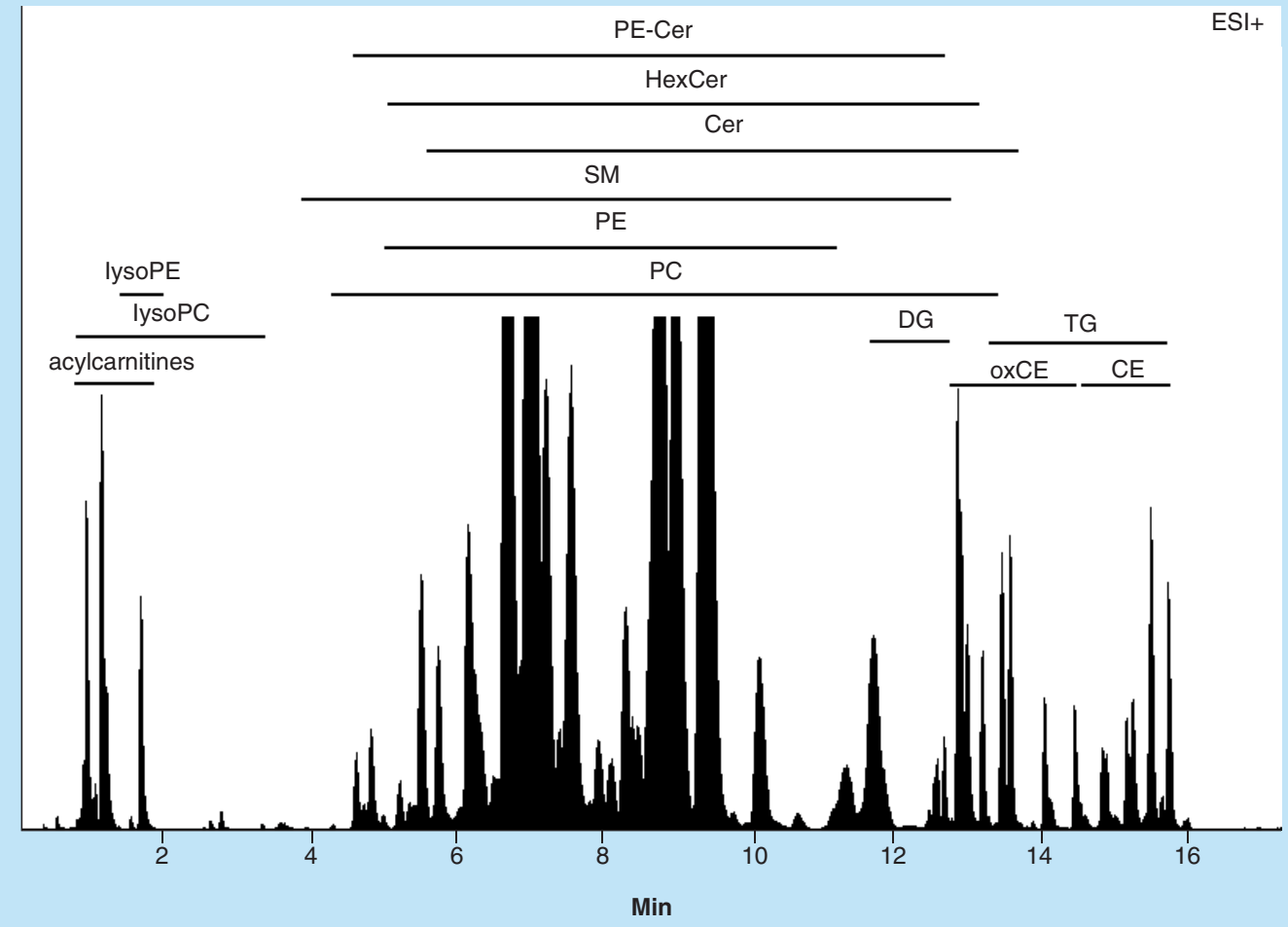

(B)

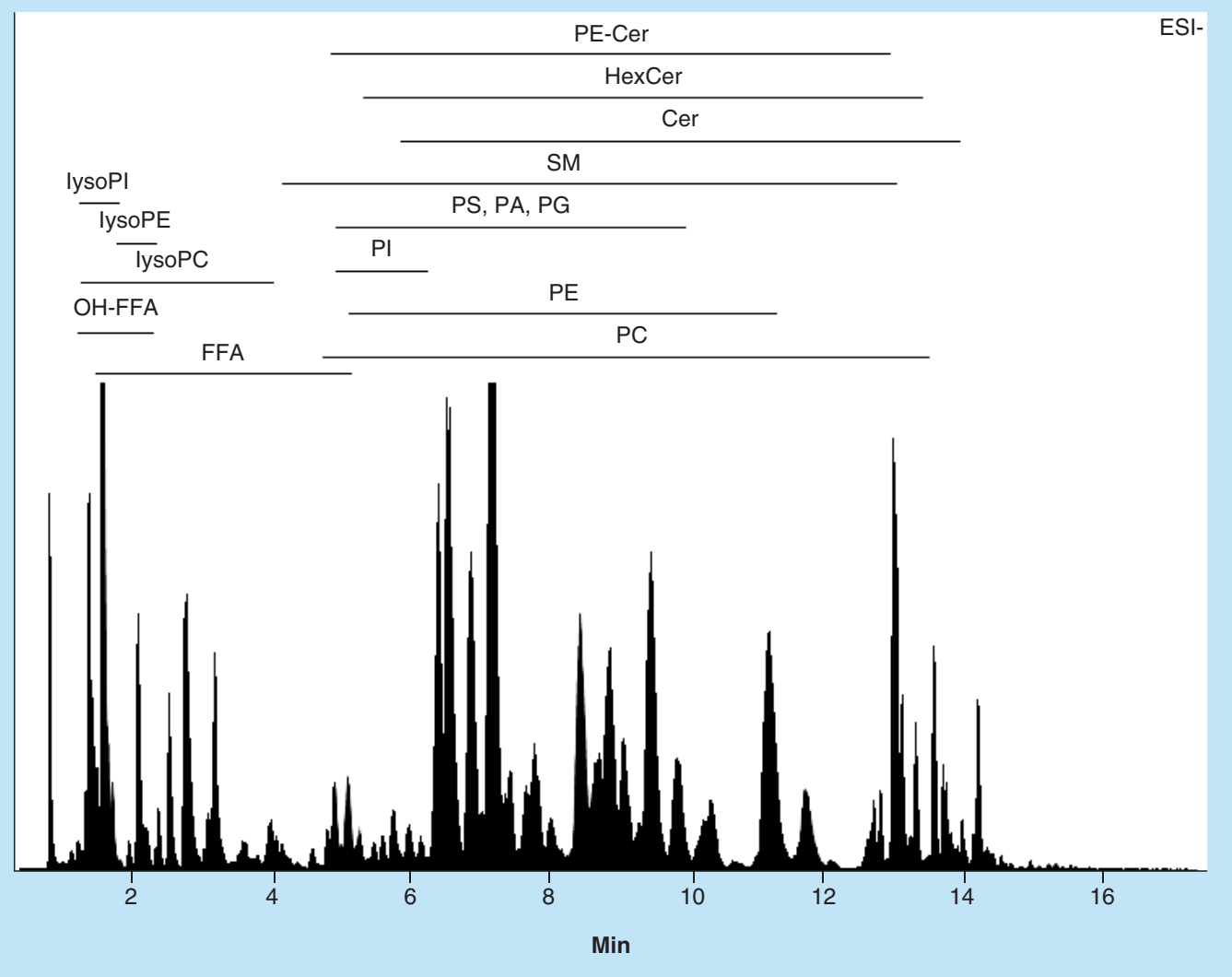

Figure 1. Typical chromatograms of human blood plasma. (A) positive and (B) negative polarity mode. The figures demonstrate the chromatographic elution time range, which designated lipid subclasses exhibit.

CE: Cholesteryl ester; Cer: Ceramide, DG: Diacylglycerol; FFA: Free fatty acid; Hex: Hexosyl; oxCE: Oxidized cholesteryl ester; PA: Phosphatidic acid; PC: Phosphatidylcholine; PE: Phosphatidylethanolamine;

PE-Cer: Ceramide-phosphoethanolamine; PG: Phosphatidylglycerol; PI: Phosphatidylinositol; PS: Phosphatidylserine SM: Sphingomyelin; TG: Triacylglycerol. 


\section{(A)}

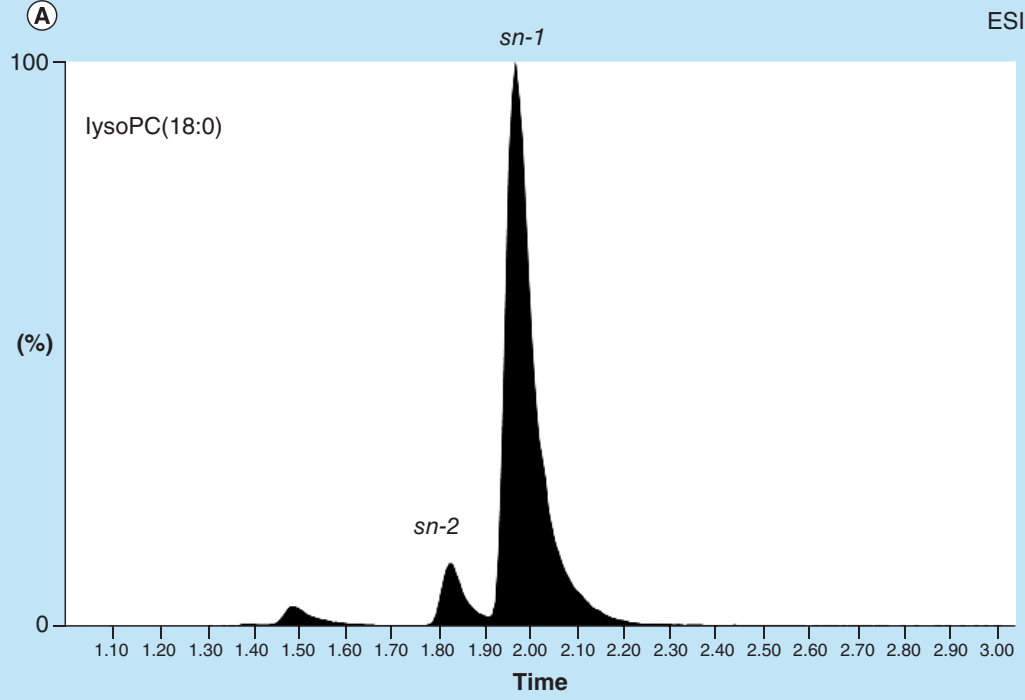

(B)

$$
\text { Time }
$$

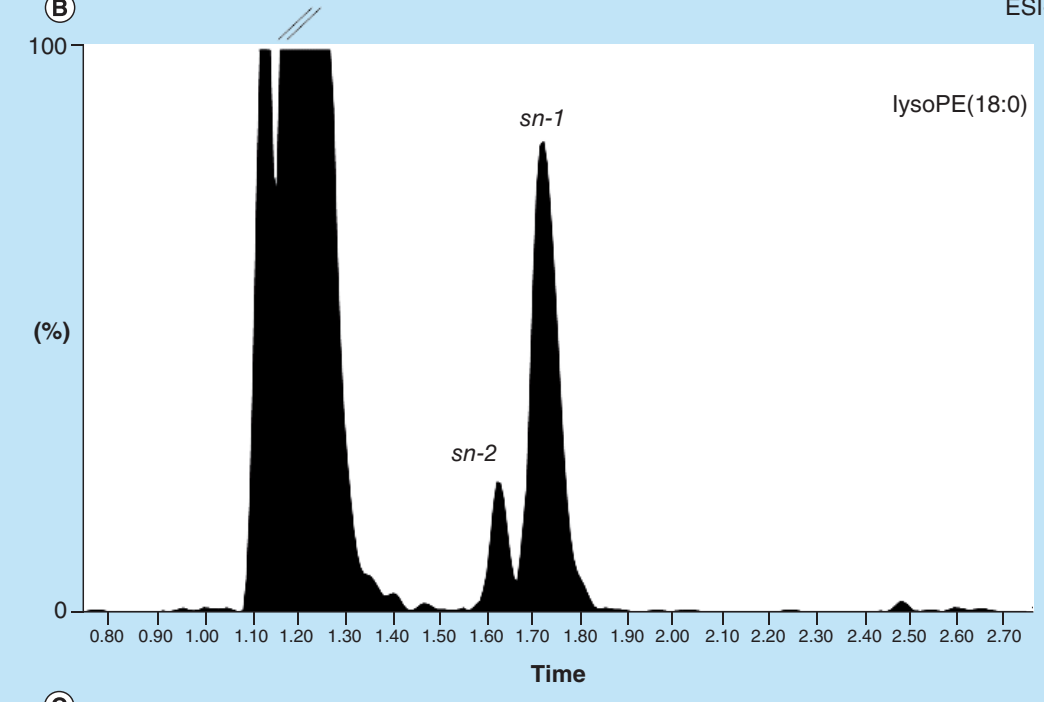

(C)

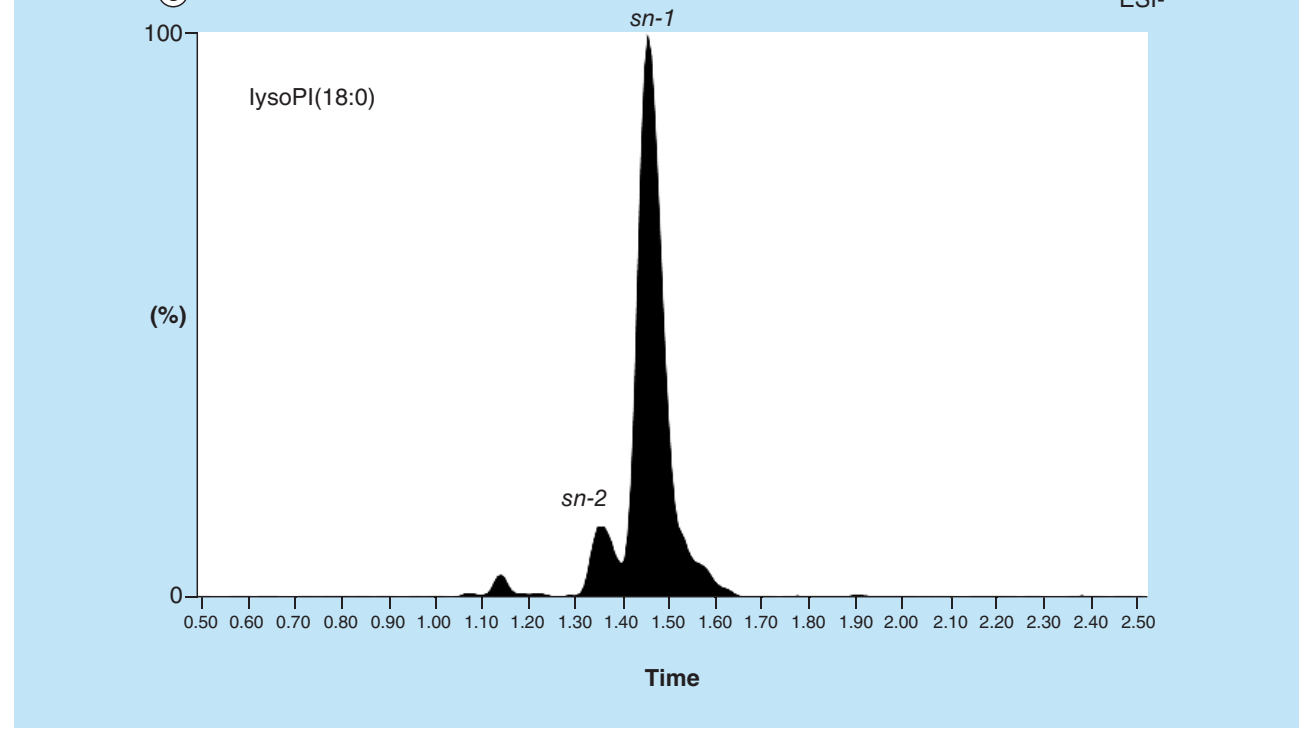

sn-1

ESI-

Figure 2. Chromatographic separation of $s n-1$ and $s n-2$ lysoglycerophospholipid isomers. Extracted ion chromatograms of (A) lysoPC(18:0) from a rat plasma extract, (B) lysoPE(18:0) from atherosclerotic plaque extracts and (C) lysoPI(18:0) from human serum extracts. The traces demonstrate the ability of the described methodology to readily resolve between $s n-1$ and $s n-2$ lyso-lipid structures.

PE: Phosphatidylethanolamine; PI: Phosphatidylinositol; PS: Phosphatidylserine. 
data in the MS mode - when MS/MS data are being acquired - and poor duty cycles. This can make DDA less attractive for the fast analysis mode of UPLC, where narrow, rapidly eluting peaks are produced [9]. However, in the context of lipid structure identification, the procedure can be aided by DDA employed in a selection of mass-range segments and in pooled, group-pooled samples and samples of extreme phenotype [18]. An example, whereby using UPLC $-M S^{\mathrm{E}}$ the arachidonoyl fragment can be readily correlated to the incorporating lipid species, can be seen in Figure 3.

\section{Lipidome coverage \& technical aspects of detected lipid classes}

It has been calculated that by combining the different constituents of each lipid (sub)class, hundreds of thousands of lipids could potentially exist [25]. The described method has demonstrated the ability for a robust and comprehensive coverage of the lipidome. The optimal performance of the method is achieved in the range of molecules with fatty chains of 10 carbons and longer. A representative list of lipids, detected with high precision, from a selection of studies is presented in Supplementary Table 1 and exceeds the total of 250 lipid molecules. A summary of the lipid categories and subclasses detected can be seen in Figure 4. The presented lipid species cover for six of the major lipid categories and 42 of the lipid subclasses. These lipid species could be assigned to 88 unique KEGG identifiers, further demonstrating the capabilities of the described approach for enhanced lipidome coverage.

Furthermore, MS acquisitions using both positive and negative polarities demonstrated the ability for detecting an expanded range of subclasses due to their preferential ionization in only one of the two polarities. The bioanalytical characteristics of some of the most frequently encountered lipid classes and subclasses are summarized in the following paragraphs.

Fatty acyls

Free FA, including straight chain saturated and unsaturated, of 10-carbon chains and longer, preferentially ionize in the negative polarity and are detected singly charged after a proton loss. They constitute the simplest lipid class and possess some of the lowest lipophilicities of known lipid species. Consequently, they are detected at the very first minutes of the lipid profiling chromatographic method.

Oxylipin molecules such as hydroxyl-fatty acids and leukotrienes can also be detected in the first $2 \mathrm{~min}$ of the negative mode after proton loss. Due to presence of the hydroxyl group they can also ionize and be detected at low intensities in positive mode.

Acylcarnitines, medium- and long-chain, can be routinely detected by the described lipid profiling method. Acylcarnitines esterified with 12-carbon fatty acyl chains and longer, can be detected in the positive polarity as protonated ions. They are eluting in the first 2 min of the chromatographic method.

\section{Glycerolipids}

Diacylglycerols are detected in positive mode as ammonium, sodium and potassium adducts and as protonated ions after neutral loss of water. They are eluting in the range of 12-13 min.

Triacylglycerols are detected as ammonium and sodium adducts. The high lipophilicity of triacylglycerols increases their partitioning to the C18 stationary phase. They are, therefore, detected in the retention time range of 13.5-16 min. Triacylglycerols with 12-carbon chains and longer have been observed (Supplementary Table 1).

\section{Glycerophospholipids \\ Lysophosphatidylcholines \& phosphatidylcholines}

Lysophosphatidylcholines (lysoPCs) and phosphatidylcholines (PCs) are detected in both positive and negative mode. In positive ionization mode, (lyso)PCs can be detected in their protonated cationic form and as sodium and potassium adducts. In negative MS polarity, (lyso)PCs are detected as formate adducts $\left([\mathrm{HCOOH}-\mathrm{H}]^{-}\right)$which is attributed to the presence of formate anions in the mobile phases (deriving from FA and ammonium formate). They can also be detected after a methyl loss from their N,N,N-trimethylethanolammonium group. The PCs typically have retention times between 4.5 and $14 \mathrm{~min}$. They are well separated from lysoPCs, which are eluting in the 0.9-3.5 min region. Importantly, the described method has the ability to chromatographically resolve $s n-1$ and $s n-2$ lysoPCs (Figure 2A). This high level of separation between $s n-1$ and $s n-2$ isomers cannot be achieved with PCs, since typically the lipophilicity of the $s n-1$ and $s n-2$ isomers can be highly comparable. The described method can readily perform for both acyl and alkyl chain (lyso)PCs. Finally, several molecules incorporating odd number chains can be detected (Supplementary Table 1). 


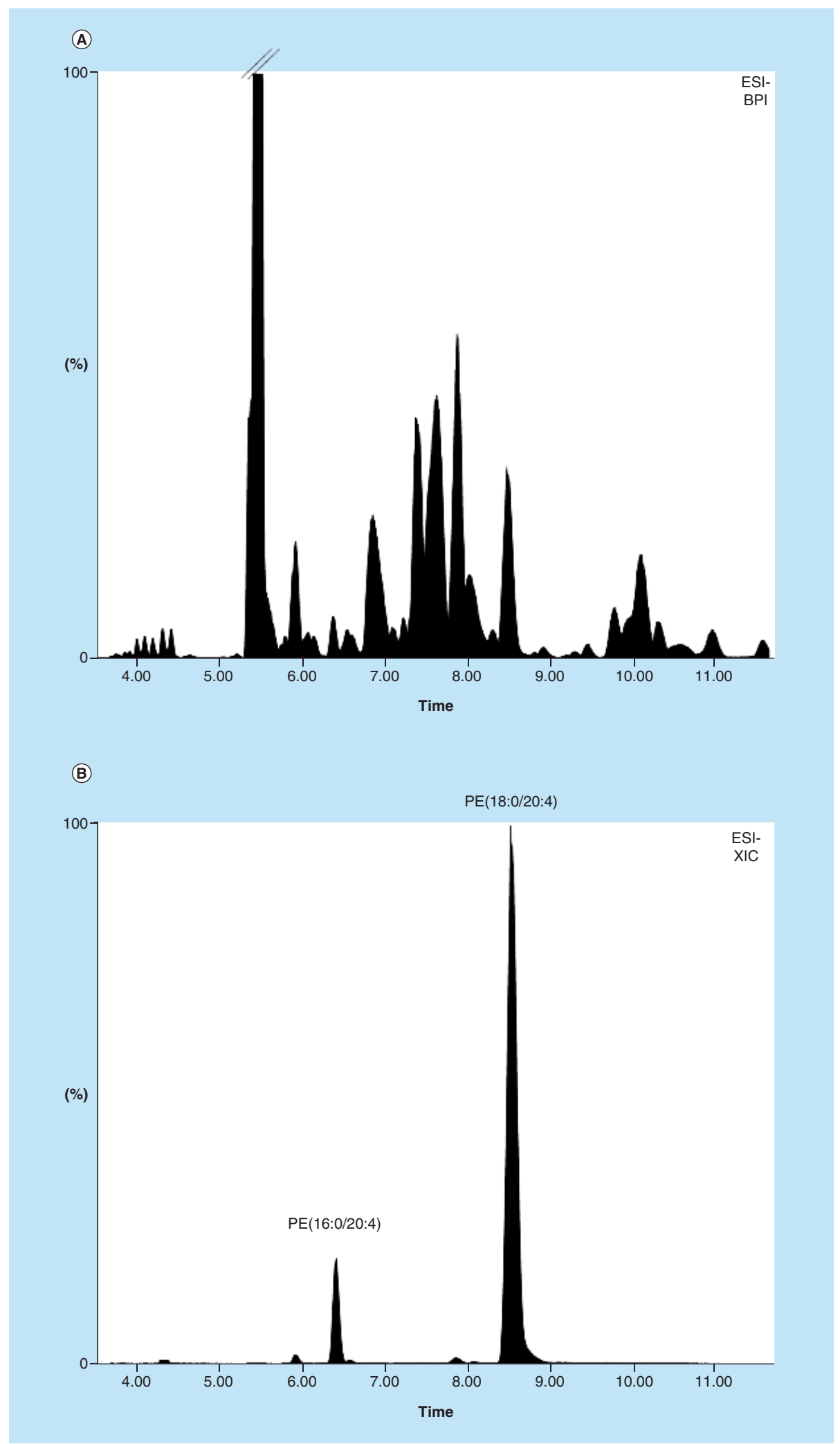

Figure 3. Correlation of extracted ion chromatograms of (data-independent) high collision energy fragments, to their parent ions. (A) Base peak intensity chromatogram (BPI) of rat plasma extract, using the described lipid profiling method. (B) Extracted ion chromatograms (XIC) of the $\mathrm{m} / \mathrm{z}$ values of the two indicated lipids, incorporating the arachidonic acyl chain in their structure. (C) Extracted ion chromatograms of m/z 303.23 Da in high collision energy mode, which is the characteristic fragment of arachidonic acyl chain. In the figure, arachidonic acyl chain fragment peaks are indicated correlating with arachidonic acyl chain incorporating lipids, both in terms of retention time and intensity.

PE: Phosphatidylethanolamine. 


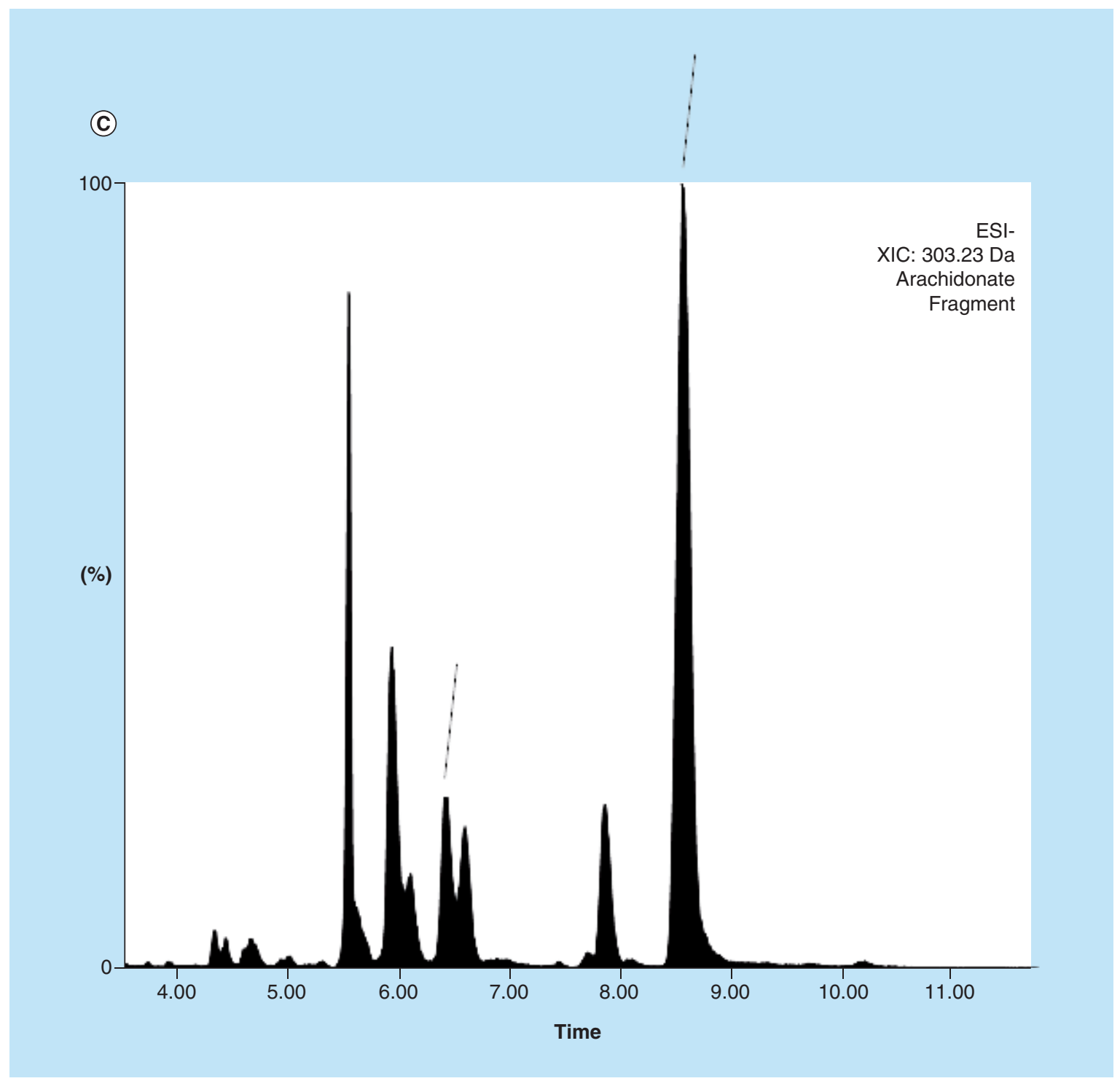

Figure 3. Correlation of extracted ion chromatograms of (data-independent) high collision energy fragments, to their parent ions (cont.). (A) Base peak intensity chromatogram (BPI) of rat plasma extract, using the described lipid profiling method. (B) Extracted ion chromatograms (XIC) of the $\mathrm{m} / \mathrm{z}$ values of the two indicated lipids, incorporating the arachidonic acyl chain in their structure. (C) Extracted ion chromatograms of $\mathrm{m} / \mathrm{z} 303.23 \mathrm{Da}$ in high collision energy mode, which is the characteristic fragment of arachidonic acyl chain. In the figure, arachidonic acyl chain fragment peaks are indicated correlating with arachidonic acyl chain incorporating lipids, both in terms of retention time and intensity. PE: Phosphatidylethanolamine.

\section{Lysophosphatidylethanolamines \& phosphatidylethanolamines}

Lysophosphatidylethanolamines (lysoPEs) are detected in the chromatographic region of 1.5-2.0 min, while phosphatidylethanolamines (PEs) in the range of 5.0-11.5 min. The $s n-1$ and $s n-2$ lysoPEs exhibit different retention times and are chromatographically resolved (Figure 2B). They are detected in positive mode as $[\mathrm{M}+\mathrm{H}]+$ and $[\mathrm{M}+\mathrm{Na}]+$ and with enhanced ionization in negative as $[\mathrm{M}-\mathrm{H}]^{-}$. Acyl and alkyl chain (lyso)PEs are routinely detected (Supplementary Table 1).

\section{Lysophosphatidylinositols \& phospatidylinositols}

Lysophosphatidylinositols (lysoPIs) are detected with retention times of 0.9-1.5 min, while phospatidylinositols (PIs) with retention times of 4.5-6.0 min. LysoPIs can also be chromatographically resolved to their $s n-1$ and $s n-2$ 


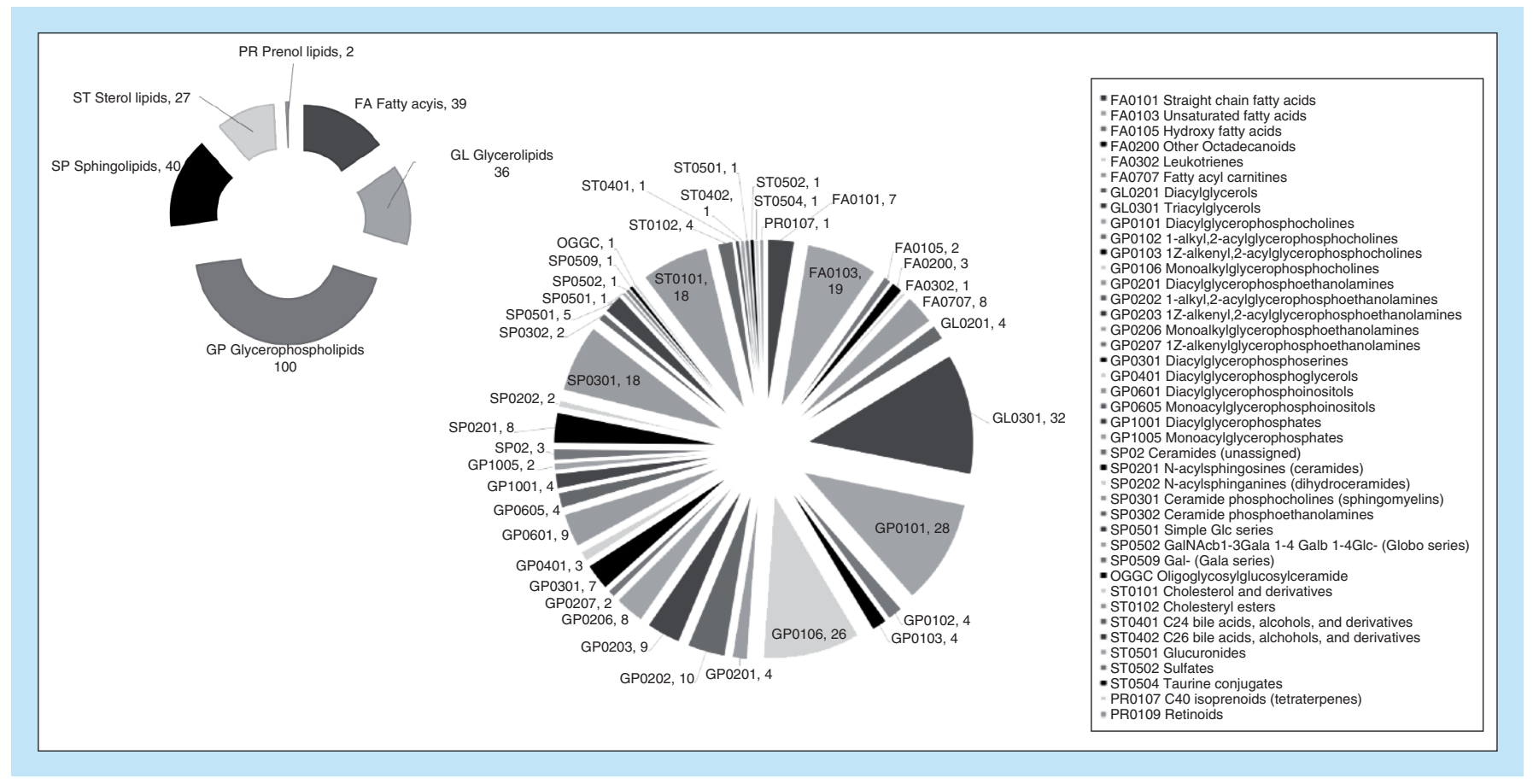

Figure 4. A schematic summarizing the lipid categories (left) and subclasses (right) detected by the described RP-UPLC-TOF-MSE approach. A detailed list of the lipids species can be found at Supplementary Table 1.

isomers (Figure 2C). Both lysoPIs and PIs preferentially ionize in negative mode. If present at high concentrations, they can be detected in very low intensities in positive MS polarity usually in a protonated form and after a loss of water.

\section{Phosphatidylserines, phosphatidic acids \& phosphatidylglycerols}

Phosphatidylserines (PSs), phosphatidic acids (PAs) and phosphatidylglycerols (PGs) are predominantly detected in negative ionization mode after proton loss. PSs can also be detected in positive mode as protonated cations. PGs can be detected in positive mode as ammonium adducts. These phospholipids are eluting in the region of 4.0-10.0 min.

\section{Sphingolipids}

The sphingolipid category is structurally characterized by an aliphatic amino alcohol backbone chain. It is also a very broad lipid class, composed by several subclasses readily detected by the described method:

Sphingomyelins (SMs) are the most abundant sphingolipid subclass, in mammals. SMs exhibit elution times spanning from 4-13.5 min. They can provide high intensity signals in both positive and negative modes. They are generally detected as protonated and sodiated adducts in positive and as formate and methyl loss (from their $\mathrm{N}, \mathrm{N}, \mathrm{N}$-trimethylethanolammonium group) adducts in negative.

Ceramides can be detected with retention times in the range of 5.5-14 min. Ionizing in both polarities, they can produce protonated cations after water loss and sodium adducts in positive mode, while they can be detected as deprotonated ions in negative mode.

Hexosylceramides including mono-, di-, tri- and tetra-hexosylceramides can be detected in the range of 5-13.5 min. In positive mode, hexosylceramides can be detected as proton and sodium adducts. In negative mode, they can form deprotonated ions and formate adducts.

Ceramide-phosphoethanolamines can be detected as protonated and sodiated ions in positive and deprotonated in negative. They are eluting between 5 and $13 \mathrm{~min}$. 


\section{Sterol lipids}

By employing the described lipid profiling method, a range of sterol lipids can be detected. First, free cholesterol could be detected in positive mode as a protonated ion, after a neutral loss of water. Furthermore, cholesteryl sulfate and cholesteryl glucuronide can be detected in the negative polarity. Cholesteryl esters (CEs) are detected in the chromatographic region of 15-16 min, while a wide range of oxidized CEs is eluting in the region of 13-15 min. Both CEs and oxidized CEs are preferentially ionizing in positive mode and as sodium and ammonium adducts. Last, bile acids can be detected in the first $2.5 \mathrm{~min}$ of the chromatographic method in negative mode. Sterol lipids that have been detected in studied biological matrices are listed in Supplementary Table 1.

\section{Prenol lipid class}

Prenol lipid subclasses such as carotenoids and retinols (vitamin A), are detected in positive mode as protonated and protonated after water loss ions. They are eluting in the range of 3-7 min.

\section{Future perspective}

The field of UPLC-MS lipidomics has a lot to gain from recent and expected future advancements in instrumentation. Already, areas of lipid research are being rediscovered, altering the way lipid species are biologically perceived. This predominantly relies on the resolving abilities of modern lipidomics tools and improved MS sensitivity of detection. However, with the current capabilities, comprehensive elucidation of lipid molecular structure cannot be yet achieved in untargeted lipidomics. The assignment of the carbon position of the glycerol molecule to which the fatty chains are esterified can be elusive, especially in di- and tri- fatty acyl chain lipids. Similarly, double-bond positioning and to an extent geometric isomerism (cis- and trans-) can be difficult to decipher. These molecular variations can be critical in disease $[26,27]$ and demand full elucidation.

A next-generation-performance liquid chromatography that could surpass the performance of UPLC could be an exciting possibility. A technique able to achieve a higher level of chromatographic resolution could deliver further separation of isobaric lipid isomers. Apart from the higher chromatographic resolution, the field could further benefit by faster turnaround times of analysis. Moreover, the separation of highly lipophilic molecules such as TGs and very long chain phospholipids is a caveat that needs to be addressed by new chromatography stationary phase chemistries. However, these advancements could take several years to be accomplished and commercialized. Therefore, novel approaches with greater improvement margins need to be promoted.

Ion mobility separation (IMS) has been used in the past few years as an additional level of separation in MS [28]. A higher resolution ability is to be expected in the near future from this technique and it is anticipated to significantly assist in the resolution of geometric isomers. However, separation by IMS will always be unachievable in lipids with similar rotationally-averaged collision cross-section. Nonetheless, it can always provide a rapid alternative to liquid chromatography by delivering an additional level of preMS separation.

Novel analytical techniques such as ozone-induced dissociation (OzID) [29] can offer an unconventional but highly effective solution to the determination of the double bond position (positional isomers). The possibility of using multi-gas delivery systems to MS components along with independent data collection, should not be excluded. The high acquisition frequencies of modern MS instruments, can assist in this task by facilitating the acquisition of multiple, parallel, alternating scans, with each independent scan using a different gas for performing collision induced dissociation.

Untargeted and semitargeted methods will continue to dominate the field of lipidomics. This goes beyond the desire for discovering novel molecules (both for involvement in disease or of previously unknown molecular structures) and subsequently satisfying the ubiquitous in science neophilia. In fact, lipid molecules have the benefit of easier molecular structure assignment process, as compared with small molecules. This process is expected to profit significantly by anticipated improvements in instrument sensitivity and performing dynamic range. Untargeted approaches should be followed through and complemented by targeted and where necessary quantitative methodologies.

Lastly, more sophisticated bioinformatics tools, able to manage the vast amount of data and report in concise and practical tables and figures, are soon to be expected. Informatics packages that can aid significantly with chemical structure annotation will further become more automated and user friendly. 
Executive summary

- The vast diversity of lipids renders their analysis in biological samples very challenging.

- RP-UPLC-MS is now the method of choice for untargeted lipidomics.

Method

- An untargeted RP-UPLC-TOF-MSE method is proposed for the comprehensive cover of lipid molecules from a wide range of categories and subclasses.

Advantages of the use of RP-UPLC

- The utilization of RP chromatography can deliver superior performance as compared with past chromatographic techniques.

- UPLC can provide higher separation depicted by resolving geometric isomers (cis-/trans-) and positional isomers (sn-1 and sn-2 positions of the fatty acyl chain, esterified to glycerol).

Advantages of the use of $\mathrm{MS}^{\mathrm{E}}$ data-independent acquisition in untargeted lipidomics

- The use of $M S^{E}$ can provide an additional level of specificity.

- A combination of $\mathrm{MS}^{\mathrm{E}}$ and MS/MS can significantly assist in lipid structure assignment.

Lipidome coverage \& technical aspects for detected lipid classes

- The described method can cover the majority of lipid classes: glycerolipid, glycerophospholipid, sphingolipid, fatty acyls, sterol lipids and prenol lipids.

- A representative list of lipids, from a selection of studies exceeds the total of 250 molecules and demonstrates the efficacy of the employed approach.

- The presented lipid species cover for 42 of the lipid subclasses and were assigned to 88 unique KEGG identifiers.

- The optimal performance of the method is achieved in the range of molecules with fatty chains of 10 carbons and longer.

\section{Future perspective}

- Despite of the recent achievements in lipid isomer separation, additional performance is required for comprehensive elucidation of lipid molecular structure.

- In the lack of advancements in chromatography, novel techniques, such as IMS and OzID, need to be integrated in the current UPLC-TOF-MSE platform to deliver the required separation.

Supplementary data

To view the supplementary data that accompany this paper, please visit the journal website at: www.futurescience.com/doi/suppl/10.4155/bio-2017-0249

Acknowledgements

The author would like to acknowledge the support of Dr Jia V Li.

Disclaimer

The views expressed in this publication are those of the author and not necessarily those of the NHS, the National Institute for Health Research or the Department of Health.

Financial \& competing interests disclosure

This report is independent research funded by the National Institute for Health Research (NIHR) Imperial Biomedical Research Center (BRC) (Grant No. P69964). The author has no other relevant affiliations or financial involvement with any organization or entity with a financial interest in or financial conflict with the subject matter or materials discussed in the manuscript apart from those disclosed.

No writing assistance was utilized in the production of this manuscript.

Open access

This work is licensed under the Attribution-NonCommercial-NoDerivatives 4.0 Unported License. To view a copy of this license, visit http://creativecommons.org/licenses/by-nc-nd/4.0/

\section{Ethical conduct of research}

The authors state that they have obtained appropriate institutional review board approval or have followed the principles outlined in the Declaration of Helsinki for all human or animal experimental investigations. In addition, for investigations involving human subjects, informed consent has been obtained from the participants involved. 


\section{References}

1. Quehenberger O, Dennis EA. The human plasma lipidome. N. Engl. J. Med. 365(19), 1812-1823 (2011).

2. Pietiläinen $\mathrm{KH}$, Róg T, Seppänen-Laakso $\mathrm{T}$ et al. Association of lipidome remodeling in the adipocyte membrane with acquired obesity in humans. PLoS Biol. 9(6), e1000623 (2011).

3. Wymann MP, Schneiter R. Lipid signalling in disease. Nat. Rev. Mol. Cell Biol. 9(2), 162-176 (2008).

4. Bismuth J, Lin P, Yao Q, Chen C. Ceramide: a common pathway for atherosclerosis? Atherosclerosis 196(2), 497-504 (2008).

5. Fahy E, Subramaniam S, Murphy RC et al. Update of the LIPID MAPS comprehensive classification system for lipids. J. Lipid Res. 50(Suppl.), S9-S14 (2009).

6. Yore MM, Syed I, Moraes-Vieira PM et al. Discovery of a class of endogenous mammalian lipids with anti-diabetic and anti-inflammatory effects. Cell 159(2), 318-332 (2014).

7. Patti GJ, Yanes O, Shriver LP et al. Metabolomics implicates altered sphingolipids in chronic pain of neuropathic origin. Nat. Chem. Biol. 8(3), 232-234 (2012).

8. Vorkas PA, Shalhoub J, Isaac G et al. Metabolic phenotyping of atherosclerotic plaques reveals latent associations between free cholesterol and ceramide metabolism in atherogenesis. J. Proteome Res. 14(3), 1389-1399 (2015).

9. Plumb RS, Johnson KA, Rainville P et al. UPLC/MS(E); a new approach for generating molecular fragment information for biomarker structure elucidation. Rapid Commun. Mass Spectrom. 20(13), 1989-1994 (2006).

10. Anwar MA, Vorkas P, Li JV, Want E, Davies AH, Holmes E. Optimization of metabolite extraction of human vein tissue for ultra performance liquid chromatography-mass spectrometry and nuclear magnetic resonance-based untargeted metabolic profiling. Analyst 140, 7586-7597 (2015).

11. Djekic D, Pinto R, Vorkas PA, Henein MY. Replication of LC-MS untargeted lipidomics results in patients with calcific coronary disease: an interlaboratory reproducibility study. Int. J. Cardiol. 222, 1042-1048 (2016).

12. Vorkas PA, Isaac G, Holmgren A et al. Perturbations in fatty acid metabolism and apoptosis are manifested in calcific coronary artery disease: an exploratory lipidomic study. Int. J. Cardiol. 197, 192-199 (2015).

13. Lamour SD, Gomez-Romero M, Vorkas PA et al. Discovery of infection associated metabolic markers in human African trypanosomiasis. PLoS Negl. Trop. Dis. 9(10), e0004200 (2015).

14. Vorkas PA, Shalhoub J, Lewis MR et al. Metabolic phenotypes of carotid atherosclerotic plaques relate to stroke risk: an exploratory study. Eur. J. Vasc. Endovasc. Surg. 52(1), 5-10 (2016).

15. Vorkas PA, Isaac G, Anwar MA et al. Untargeted UPLC-MS profiling pipeline to expand tissue metabolome coverage: application to cardiovascular disease. Anal. Chem. 87(8), 4184-4193 (2015).

16. Anwar MA, Adesina-Georgiadis KN, Spagou K et al. A comprehensive characterisation of the metabolic profile of varicose veins; implications in elaborating plausible cellular pathways for disease pathogenesis. Sci. Rep. 7(1), 2989 (2017).

17. Anwar MA, Vorkas PA, Li J et al. Prolonged mechanical circumferential stretch induces metabolic changes in rat inferior vena cava. Eur. J. Vasc. Endovasc. Surg. 52(4), 544-552 (2016).

18. Vorkas PA, U MRA, Li JV. Tissue multiplatform-based metabolomics/metabonomics for enhanced metabolome coverage. Methods Mol. Biol. (2018) (In press).

19. Smith CA, Want EJ, O'maille G, Abagyan R, Siuzdak G. XCMS: processing mass spectrometry data for metabolite profiling using nonlinear peak alignment, matching and identification. Anal. Chem. 78(3), 779-787 (2006)

20. Smith CA, O'maille G, Want EJ et al. METLIN: a metabolite mass spectral database. Ther. Drug Monit. 27(6), 747-751 (2005).

21. Wishart DS, Knox C, Guo AC et al. HMDB: a knowledgebase for the human metabolome. Nucleic Acids Res. 37, D603-D610 (2009).

22. Fahy E, Sud M, Cotter D, Subramaniam S. LIPID MAPS online tools for lipid research. Nucleic Acids Res. 35, W606-W612 (2007).

23. Wilson ID, Nicholson JK, Castro-Perez J et al. High resolution 'ultra performance' liquid chromatography coupled to oa-TOF mass spectrometry as a tool for differential metabolic pathway profiling in functional genomic studies. J. Proteome Res. 4(2), 591-598 (2005).

24. Want EJ, Wilson ID, Gika $\mathrm{H}$ et al. Global metabolic profiling procedures for urine using UPLC-MS. Nat. Protoc. 5(6), 1005-1018 (2010).

25. Yetukuri L, Ekroos K, Vidal-Puig A, Orešič M. Informatics and computational strategies for the study of lipids. Mol. Biosyst. 4(2), 121-127 (2008).

26. Lavi S, Mcconnell JP, Rihal CS et al. Local production of lipoprotein-associated phospholipase A2 and lysophosphatidylcholine in the coronary circulation: association with early coronary atherosclerosis and endothelial dysfunction in humans. Circulation 115(21), 2715-2721 (2007).

27. Guijas C, Meana C, Astudillo AM, Balboa MA, Balsinde J. Foamy monocytes are enriched in cis-7-hexadecenoic fatty acid (16:1n-9), a possible biomarker for early detection of cardiovascular disease. Cell Chem. Biol. 23(6), 689-699 (2016).

28. Guevremont R, Siu KW, Wang J, Ding L. Combined ion mobility/time-of-flight mass spectrometry study of electrospray-generated ions. Anal. Chem. 69(19), 3959-3965 (1997). 
29. Thomas MC, Mitchell TW, Harman DG, Deeley JM, Nealon JR, Blanksby SJ. Ozone-induced dissociation: elucidation of double bond position within mass-selected lipid ions. Anal. Chem. 80(1), 303-311 (2008). 
